\title{
Profiling of Polar Lipids in Marine Oleaginous Diatom Fistulifera solaris JPCC DA0580: Prediction of the Potential Mechanism for Eicosapentaenoic Acid-Incorporation into Triacylglycerol
}

\author{
Yue Liang ${ }^{1}$, Yoshiaki Maeda ${ }^{1}$, Tomoko Yoshino ${ }^{1}$, Mitsufumi Matsumoto ${ }^{2,3}$ and \\ Tsuyoshi Tanaka ${ }^{1,3}, *$
}

1 Division of Biotechnology and Life Science, Institute of Engineering, Tokyo University of Agriculture and Technology, 2-24-16, Naka-cho, Koganei, Tokyo 184-8588, Japan;

E-Mails: y_liang@cc.tuat.ac.jp (Y.L.); y_maeda@cc.tuat.ac.jp (Y.M.); y-tomoko@cc.tuat.ac.jp (T.Y.)

2 Biotechnology Laboratory, Electric Power Development Co., Ltd., 1, Yanagisaki-machi, Wakamatsu-ku, Kitakyusyu 808-0111, Japan; E-Mail: mitsufumi_matsumoto@jpower.co.jp

3 Japan Science and Technology Agency (JST), Core Research for Evolutionary Science and Technology (CREST), 5, Sanbancho, Chiyoda-ku, Tokyo 102-0075, Japan

* Author to whom correspondence should be addressed; E-Mail: tsuyo@cc.tuat.ac.jp; Tel.: +81-423-887-401; Fax: +81-423-857-713.

Received: 20 February 2014; in revised form: 22 April 2014 / Accepted: 23 April 2014 / Published: 28 May 2014

\begin{abstract}
The marine oleaginous diatom Fistulifera solaris JPCC DA0580 is a candidate for biodiesel production because of its high lipid productivity. However, the substantial eicosapentaenoic acid (EPA) content in this strain would affect the biodiesel quality. On the other hand, EPA is also known as the essential health supplement for humans. EPAs are mainly incorporated into glycerolipids in the microalgal cell instead of the presence as free fatty acids. Therefore, the understanding of the EPA biosynthesis including the incorporation of the EPA into glycerolipids especially triacylglycerol (TAG) is fundamental for regulating EPA content for different purposes. In this study, in order to identify the biosynthesis pathway for the EPA-containing TAG species, a lipidomic characterization of the EPA-enriched polar lipids was performed by using direct infusion electrospray ionization (ESI)-Q-TRAP-MS and MS/MS analyses. The determination of the fatty acid positional distribution showed that the $s n-2$ position of all the chloroplast lipids and part of phosphatidylcholine (PC) species was occupied by C16 fatty acids. This result
\end{abstract}


suggested the critical role of the chloroplast on the lipid synthesis in $F$. solaris. Furthermore, the exclusive presence of $\mathrm{C} 18$ fatty acids in PC highly indicated the biosynthesis of EPA on PC. Finally, the PC-based acyl-editing and head group exchange processes were proposed to be essential for the incorporation of EPA into TAG and chloroplast lipids.

Keywords: marine oleaginous diatom; Fistulifera solaris; lipid synthesis; eicosapentaenoic acid (EPA); PC-based acyl-editing

\section{Introduction}

Over the past several decades, biofuel production has attracted much interest due to the approaching exhaustion of fossil fuels and their negative impacts on climate. Microalgae have been recognized as promising biofuel producers due to their advantages of higher biomass productivity and non-competition with foods [1-3]. Recent studies have discovered a number of microalgae possessing high content of lipids [4-9].

In addition to lipid content, the unsaturation degree of microalgal lipids, which directly determines the fuel quality, is another critical factor for biodiesel application [10]. High content of polyunsaturated fatty acid (PUFA) will result in low oxidative stability of the final product [11]. The PUFA ( $\geq 4$ double bonds) content in biodiesel is limited to less than $1 \%$ by the EN 14214 standards in Europe. However, on the other hand, omega 3-long chain PUFAs (133-LCPUFAs), e.g., eicosapentaenoic acid (EPA, C20:5n3) and docosahexaenoic acid (DHA, C22:5n3) are essential health supplements for humans [12]. The growing market of $\omega 3$-LCPUFAs has made it impossible to maintain a sustainable supply from the conventional source of fish oil. Therefore, the research in polyunsaturated lipid synthesis in microalgae thus receives much attention. Some microalgal strains, which were determined to produce high content of EPA and DHA [13], are the potential alternative source of $\omega 3$-LCPUFAs. The understanding of the biosynthesis pathway for $\omega 3$-LCPUFA and the assembly of $\omega 3$-LCPUFA into lipids is the essential task to control $\omega 3$-LCPUFA content in microalgae by means of metabolic engineering.

Among eukaryotic microalgae, a pennate diatom, Phaeodactylum tricornutum has been used as one of the model organisms for studies in $\omega 3$-LCPUFA synthesis $[14,15]$ due to its high content of EPA [16], available genome information [17] and easy transformation [18,19]. In P. tricornutum, metabolic labeling analyses revealed that phosphatidylcholine (PC) was involved in the series of fatty acid-desaturation for EPA synthesis [15]. However, the mechanism how EPA is incorporated into triacylglycerol (TAG, a main source of biodiesel) has not yet thoroughly been discussed in any microalgae. Furthermore, the lipid content in P. tricornutum is moderate [5]; thus, this microalga may not be the best lipid producer for biodiesel production. In addition, the knowledge of polyunsatulated lipid synthesis in other diatoms is poorly accumulated.

In this study, we focused on another marine pennate diatom, Fistulifera solaris (formerly Fistulifera sp.) JPCC DA0580, which has been identified in our lab [20] as an oleaginous microalga and can accumulate oil up to nearly $60 \%$ of dried cell weight (DCW) [9,21]. F. solaris also maintains substantial amount of EPA [9,22]. Owing to the achievements on mass cultivation [9,23] genome 
sequencing (partially published [24]) and genetic transformation [25], we propose that this strain is one of the ideal candidates for either biofuel or EPA production. Recently, the lipidomic analysis targeting neutral lipids, and the desaturation process for EPA synthesis in F. solaris has been reported [22,26], while the exact substrates for desaturation and the process of EPA-incorporation into TAG have not yet been addressed. In this study, the fatty acid composition and positional distribution of major polar lipid species were determined. The distribution of EPA and its precursor fatty acids in polar lipids was analyzed with direct infusion electrospray ionization (ESI)-Q-TRAP-MS/MS in order to deeply understand the polyunsatulated lipid synthesis in $F$. solaris. The results suggest that EPA was desaturated on PC and the PC-based acyl-editing and head group exchange processes may contribute to the EPA-incorporation into TAG.

\section{Results and Discussion}

\subsection{Polar Lipid Profile of Fistulifera solaris JPCC DA0580}

In order to examine how EPA is synthesized and incorporated into TAGs in $F$. solaris, the polar lipid profile including the information of fatty acid composition and positional distribution was elucidated by direct infusion ESI-Q-TRAP-MS/MS, through which the intact molecular structures of lipids can be determined. In the phospholipid (PL) fraction, 14 types of lipids including 11 phosphatidylcholines (PCs), 2 phosphatidylglycerol (PGs), and 1 phosphatidylinositol (PI) were identified (Figure 1). Phosphatidylethanolamine (PE) was not detected in the PL fraction, indicating the absence of PE in $F$. solaris. In the glycolipid (GL) fraction, 16 types of lipids including 8 monogalactosyldiacylglycerol (MGDGs), 5 digalactosyldiacylglycerol (DGDGs), and 3 sulfoquinovosyldiacylglycerol SQDGs were identified (Figure 2).

There are two distinct pathways for glycerolipid assembly in higher plants [27] and microalgae [28], i.e., a prokaryotic pathway and a eukaryotic pathway. In diatoms, the chloroplast is enveloped with four membranes (outermost, second outermost, second innermost and innermost membranes, see also Figure 3). The innermost and second innermost membranes are actually corresponding to the two membranes of the primary chloroplast where glycerolipids are synthesized through the prokaryotic pathway. The space between the second outermost membrane and the outermost membrane represents the endoplasmic reticulum (ER, also known as chloroplast-ER; CER) where the eukaryotic pathway is responsible for the lipid synthesis. The lipids assembled through the prokaryotic pathway have a C16 fatty acid at the sn-2 position, while those through the eukaryotic pathway have a C18 or C20 fatty acid at the $s n-2$ position.

In $F$. solaris, the $s n-2$ position of major MGDGs, DGDGs, SQDGs, PGs and PI species was exclusively occupied by a C16 fatty acid. A part of PC species also possessed a C16 fatty acid at the $s n-2$ position (Figure 1A). In addition, the $s n-2$ position in most TAGs was also occupied by C16 fatty acids [22]. This specific distribution of $\mathrm{C} 16$ at the $s n-2$ position in large proportion of lipids was also reported in P. tricornutum [29,30]. These results indicate that the prokaryotic pathway in the chloroplast plays an essential role in the storage lipid TAG and chloroplast lipids including MGDG, DGDG, SQDG, and PG in diatoms. PC had both C16 and C18 (C20) fatty acids at the sn-2 position (Figure 1A), suggesting that both prokaryotic (in the chloroplast) and eukaryotic (in the ER) pathways could 
contribute to PC synthesis. This characteristic feature of PC has previously been confirmed only in $P$. tricornutum [30], and has not been found in higher plants and green algae, implying that this duplicated origin of PC could be a unique for diatoms. Furthermore, an F. solaris-specific feature was also found. The eukaryotic type of GLs which have been found in P. tricornutum [30] (e.g., MGDG 20:5/18:4, MGDG 20:5/20:5, MGDG 20:5/18:2, SQDG 20:5/18:4 and SQDG 22:6/18:1 (sn-1/sn-2)), were not detectable in this study.

Figure 1. The representative MS spectra of phosphatidylcholine (PC) (A); phosphatidylglycerol (PG) (B), and phosphatidylinositol (PI) (B) profile of Fistulifera sp. Fatty acids in the $s n-2$ position are represented in bold and red.

(A)
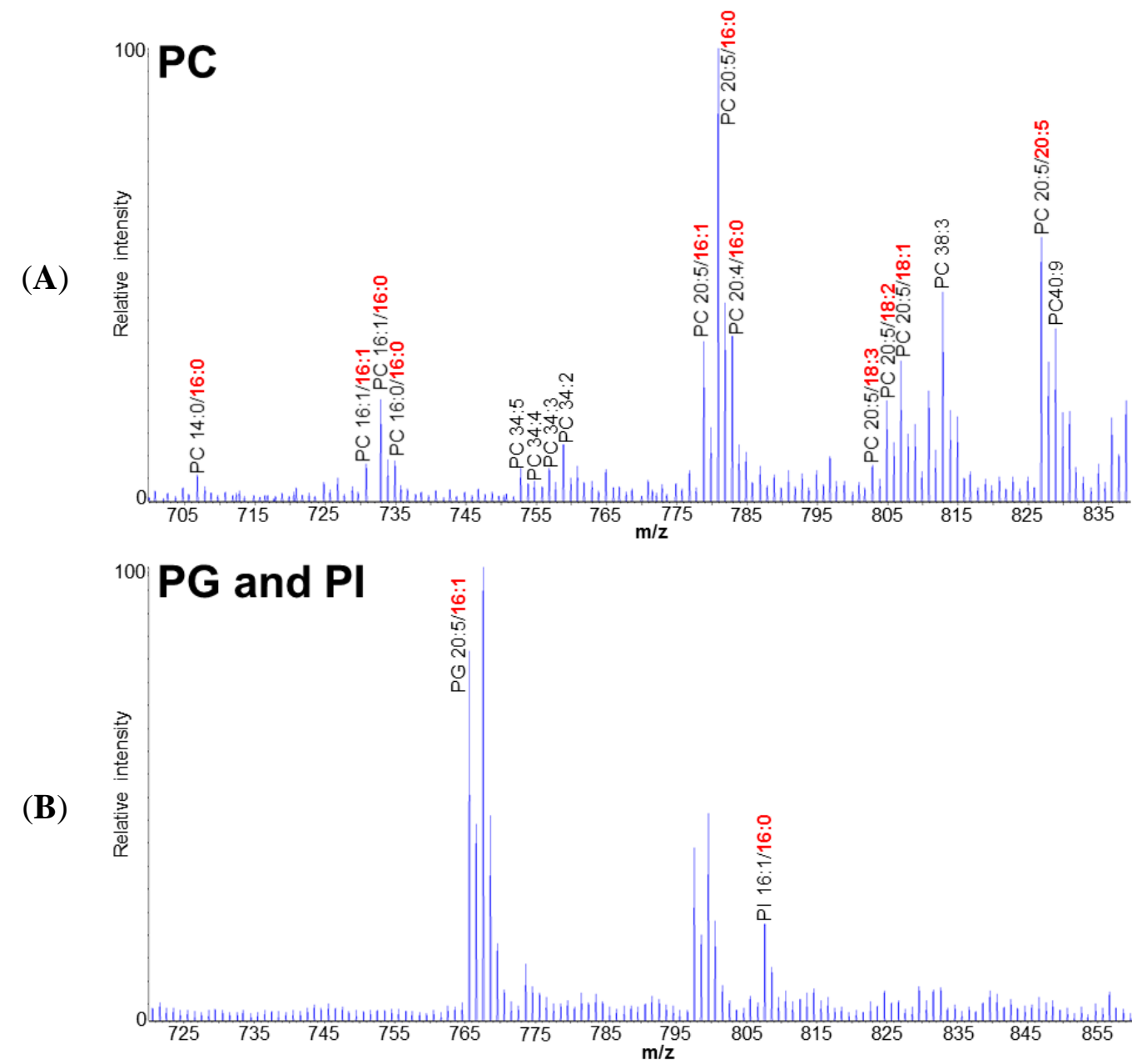

\subsection{Putative Pathways for the PUFA Synthesis in Fistulifera solaris}

Another important finding of our analysis is that C18 fatty acids (e.g., C18:1, C18:2 and C18:3) were found only on PCs (Figure 1A). More specifically, C18 fatty acids are distributed only at the $s n-2$ position of PCs, indicating that these PCs are assembled through the eukaryotic pathway in ER. Since these C18 fatty acids are essential precursors for EPA synthesis [26], we propose that EPA synthesis in F. solaris was, at least partially, performed on PC in ER (Figure 3). EPA synthesis in ER is consistent with our previous study where most enzymes involved in the EPA synthesis are localized in the ER [26,31]. Furthermore, the involvement of PC in the EPA synthesis is also consistent with the 
previous metabolic labeling study on $P$. tricornutum [15], in which it was demonstrated that the C18:1 was desaturated on $\mathrm{PC}$ to $\mathrm{C} 18: 3$ or $\mathrm{C} 18: 4$, the subsequent elongation of the $\mathrm{C} 18: 3$ and $\mathrm{C} 18: 4$ to the C20:3 and C20:4 occurred on fatty acid-CoA, and finally, the C20:3 and C20:4 were desaturated to EPA on PC. These results suggest that diatoms may share the EPA synthesis mechanism in which PCs play a central role, although we have previously demonstrated that the EPA synthesis pathway in F. solaris was simpler than in P. tricormutum [26].

Figure 2. The representative MS spectra of the monogalactosyldiacylglycerol (MGDG) (A); digalactosyldiacylglycerol (DGDG) (B); and sulfoquinovosyldiacylglycerol (SQDG) (C) profile of Fistulifera sp. Fatty acids in the $s n-2$ position are represented in bold and red.

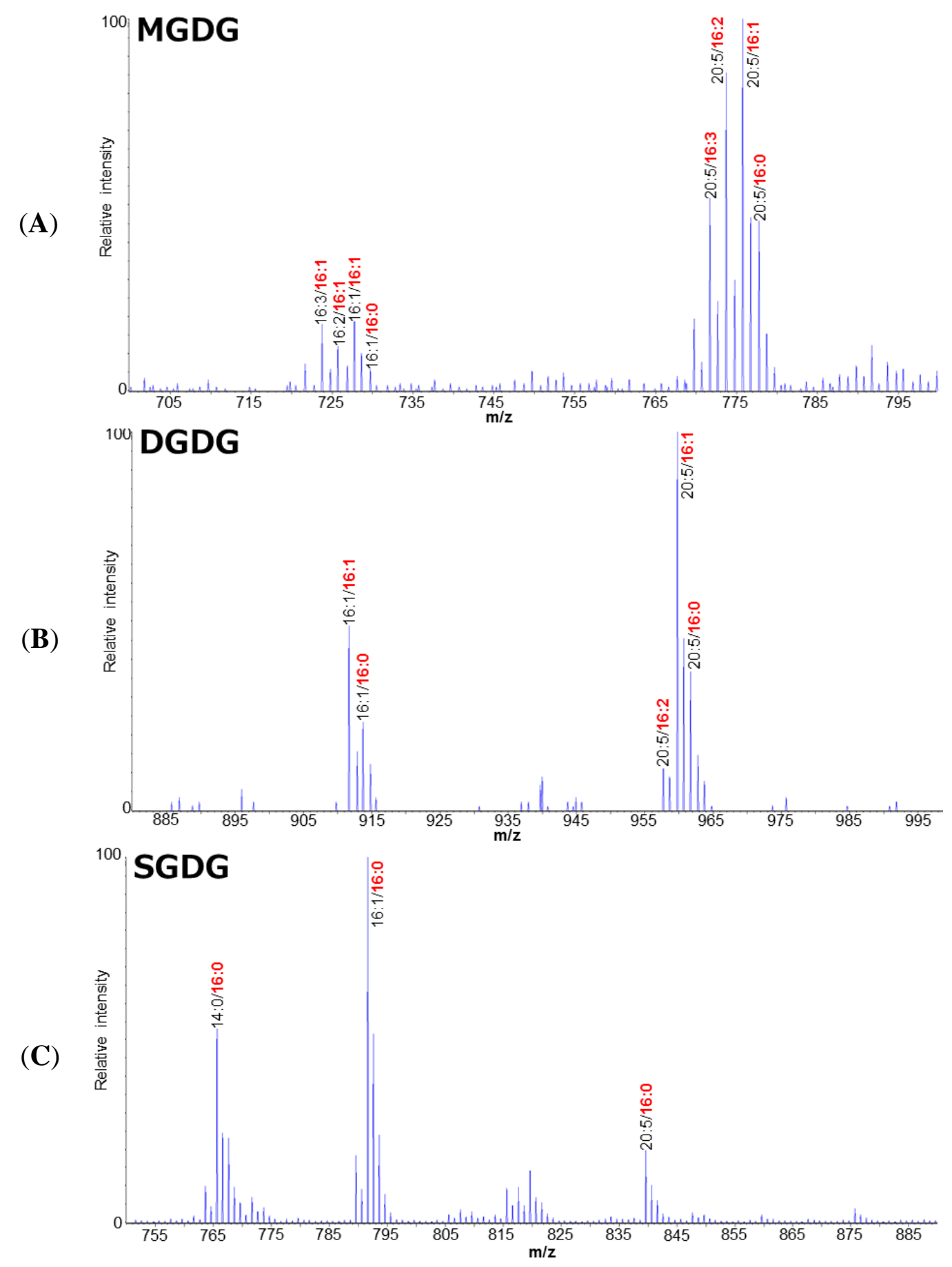


Figure 3. The scheme of the putative polar lipids and eicosapentaenoic acid (EPA) synthesis pathway in Fistulifera solaris. The plastid in this strain is considered to be surrounded by four membranes. Fatty acids and the prokaryotic lipids are de novo synthesized in the primary plastid. The eukaryotic lipids are de novo synthesized in the endoplasmic reticulum. The EPA was synthesized through the sequential desaturation of C18 fatty acid on PC. The acyl-editing and head group exchange processes on PC could not only provide EPA-CoA and EPA containing DAGs for the synthesis of other EPA-containing lipids but also promote the synthesis of the PC species maintain two C16 fatty acids. TAG in this figure is specified as the EPA-containing prokaryotic TAG. Arrow-headed lines represent synthesis, while dashed arrow-headed line represents transportation. OB, oil body; PS, periplastidal space; FAS, fatty acid synthesis; PLA, phospholipase A; LPCAT, acyl-CoA, lyso-phosphatidylcholine acyltransferase; PDCT, phosphatidylcholine diacylglycerol cholinephosphotransferase; FFA, free fatty acid; PDAT, phospholipid, diacylglycerol actyltransferase; DGAT, acyl-CoA, diacylglycerol acyltransferase; ELO, elongase.

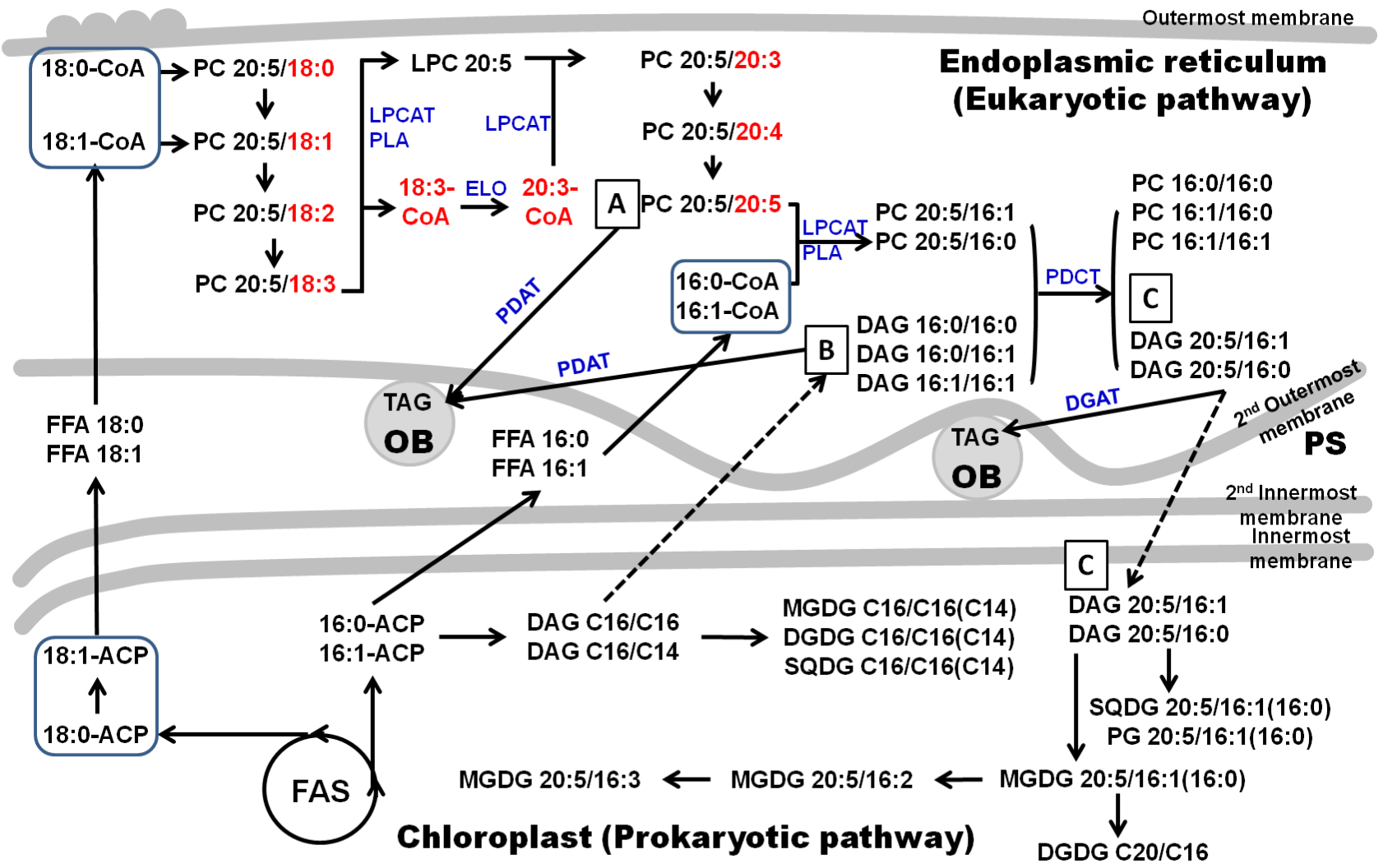

\subsection{EPA Incorporation into TAG through the Acyl-Editing Process on PC}

The EPA-containing TAGs in $F$. solaris mainly contain a $\mathrm{C} 16$ fatty acid at the sn-2 position which suggests the prokaryotic origination of those TAGs [22]. These TAGs can be theoretically synthesized by two pathways: the diacylglycerol acyltransferase (DGAT)-mediated de novo synthesis (the so called Kennedy pathway) and the phospholipid diacylglycerol acyltransferase (PDAT)-mediated membrane conversion. The DGAT catalyzes the acyltransfer reaction which incorporates the fatty acid at the fatty acid-CoA to the $s n-3$ or $s n-1$ position of the diacylglycerol (DAG) to generate TAG, while the PDAT 
catalyzes the acyltransfer reaction that incorporates the fatty acid at the $s n-2$ position of PC to the $s n-3$ or sn-1 position of DAG to generate TAG. For both enzymes, the substrate DAG is used as the backbone for TAG synthesis. In F. solaris, the exclusive existence of C14, C16 and C20:5 in the EPA-containing TAGs [22] strongly indicated that the major precursors for the synthesis of these specific TAGs could be DAG C16/C16 (sn-1/sn-2) (Figure 3; compound B) or DAG EPA/C16 (sn-1/sn-2) (Figure 3; compound $\mathrm{C}$ ) because $\mathrm{C} 14$ fatty acid presented in a minor percentage in this strain.

The most simple and reasonable pathway for the synthesis of EPA-containing prokaryotic TAG could be the PDAT-mediated acyltransfer with DAG C16/C16 (Figure 3; compound B) and PC EPA/EPA (Figure 3; compound A). Another possible pathway is DGAT-mediated acyltransfer with DAG EPA/C16 (sn-1/sn-2) (Figure 3; compound C) and fatty acid-CoA. We believe these two pathways are major contributors to incorporate EPA into TAG.

Thus far, the mechanism for the DAG EPA/C16 synthesis has never been discussed in any microalgae, but is important to be elucidated for controlling EPA-incorporation into TAG. One possibility is the de novo synthesis of DAG EPA/C16. In this mechanism, the EPA can be released from the PC in the ER to generate EPA-CoA. The generated EPA-CoA could then be transported into the chloroplast to generate EPA-ACP. Finally, the EPA on EPA-ACP is incorporated into DAG EPA/C16 in the chloroplast. However, this circuitous pathway can hardly be considered as reasonable. Therefore, we propose another pathway for the synthesis of DAG EPA/C16; i.e., the PC-based acyl-editing and head group exchange processes, which are recently demonstrated in higher plants to be essential for the incorporation of PUFA into TAG [32,33]. EPA-incorporation through this process is summarized in Figure 3. Three key enzymes are involved in these processese, the phospholipase A (PLA), acyl-CoA: lyso-phosphatidylcholine acyltransferase (LPCAT), and the phosphatidylcholine diacylglycerol cholinephosphotransferase (PDCT). The PLA catalyzes the reaction to degrade the PC into a lyso-PC (LPC) and the free fatty acid which was then converted to acyl-CoA by acyl-CoA synthetases. LPCAT catalyzes the esterification of the $s n-2$ position of a lyso-PC (LPC) to generate $\mathrm{PC}$, as well as the reverse reaction [33]. PDCT catalyzes the transfer of the phosphocholine head group from PC to DAG to generate new species of PC and DAG. The eukaryotic type of PCs which maintain an EPA at the $s n-1$ position and a $\mathrm{C} 18$ or $\mathrm{C} 20$ fatty acid at the $s n-2$ position, which were abundantly detected (Figure 1A), can be served as the starting materials to produce DAG EPA/C16. The $s n-2$ positions of these EPA-containing eukaryotic PCs are then converted to the most abundant C16 through the PLA and LPCAT mediated acyl-editing process (Figure 4, Reaction I). The generated EPA-containing PCs could subsequently transfer their head group to the predominant DAG C16/C16 through the PDCT mediated head group exchange process to generate the DAG EPA/C16 (sn-1/sn-2) and PC C16/C16 (Figure 4, Reaction II). The DAG EPA/C16 (sn-1/sn-2) can be utilized for the generation of EPA-containing TAGs as well as the EPA-containing prokaryotic MGDG, DGDG, SQDG, PG, and PI (Figure 4). 
Figure 4. The scheme of the proposed PC-based acyl-editing and head group exchange processes, which are catalyzed by PLA, LPCAT, and PDCT, for the synthesis of prokaryotic EPA-containing TAG, MGDG, DGDG, SQDG, PG and PI in F. solaris.
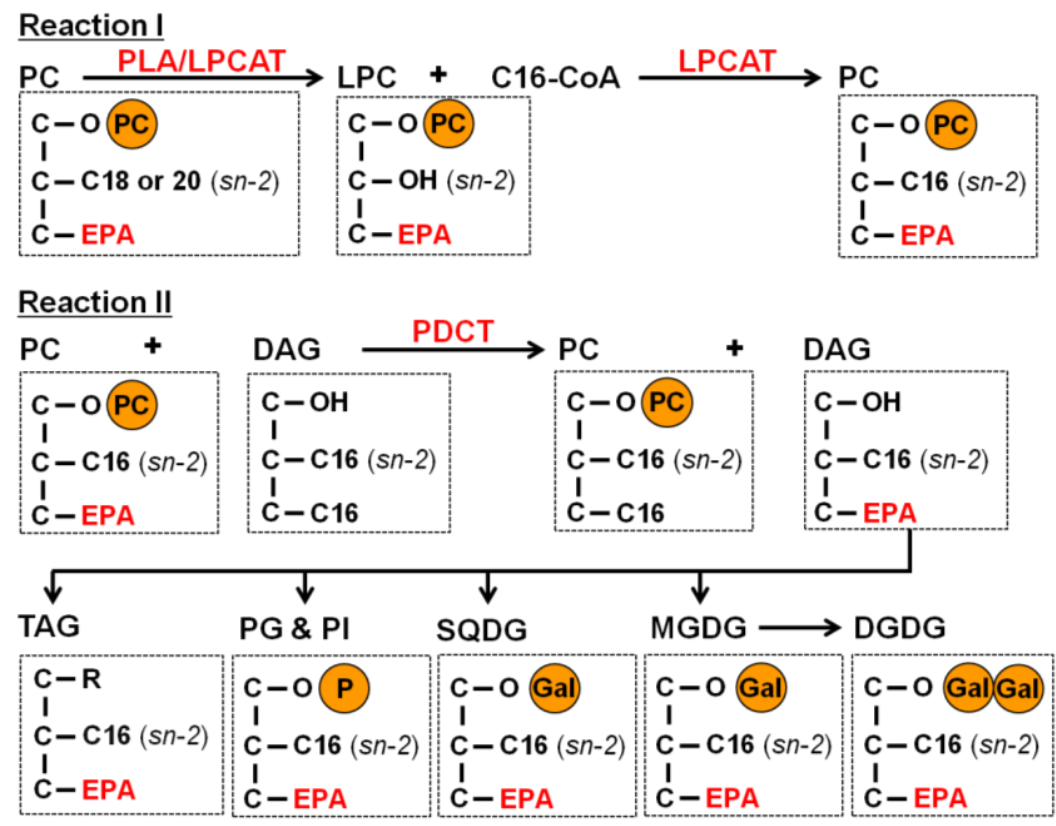

\section{Experimental Section}

\subsection{Materials}

The marine oleaginous diatom, Fistulifera sp. JPCC DA0580 was isolated by and identified in our group [21]. Silica gel 60 pre-coated thin-layer chromatography plates were from Merck (Darmstadt, Germany). The Supelclean ${ }^{\mathrm{TM}}$ LC-Si SPE Tube $(100 \mathrm{mg}, 1 \mathrm{~mL})$ was purchased from Sigma Aldrich (Tokyo, Japan). LC-MS grade methanol, LC-MS grade formic acid, and HPLC grade chloroform were purchased from Wako (Tokyo, Japan). All other reagents were of the highest commercial grade available.

\subsection{Strain and Culture Conditions}

The cells were cultured as described previously [22]. In order to enhance the polar lipids recovery in F. solaris JPCC DA0580, the nutrition-rich 10f medium which contained 10-fold more nutrition components than f medium $\left(150 \mathrm{mg} \mathrm{NaNO}, 12 \mathrm{mg} \mathrm{Na} 2 \mathrm{HPO}_{4} \cdot 2 \mathrm{H}_{2} \mathrm{O}, 1 \mu \mathrm{g}\right.$ vitamin $\mathrm{B}_{12}, 1 \mu \mathrm{g}$ biotin, $200 \mu \mathrm{g}$ thiamine $\mathrm{HCl}, 20 \mathrm{mg} \mathrm{Na} \mathrm{SiO}_{3} \cdot 9 \mathrm{H}_{2} \mathrm{O}, 8.8 \mathrm{mg} \mathrm{Na} \mathrm{NaDTA}_{2} 6.32 \mathrm{mg} \mathrm{FeCl} \cdot 6 \mathrm{H}_{2} \mathrm{O}, 24 \mu \mathrm{g}$ $\mathrm{CoSO}_{4} \cdot 5 \mathrm{H}_{2} \mathrm{O}, 42 \mu \mathrm{g} \mathrm{ZnSO} \cdot 7 \mathrm{H}_{2} \mathrm{O}, 0.36 \mathrm{mg} \mathrm{MnCl}_{2} \cdot 4 \mathrm{H}_{2} \mathrm{O}, 140 \mu \mathrm{g} \mathrm{CuSO} \cdot 5 \mathrm{H}_{2} \mathrm{O}$, and $14 \mu \mathrm{g}$ $\mathrm{Na}_{2} \mathrm{MoO}_{4} \cdot 2 \mathrm{H}_{2} \mathrm{O}$ per liter of artificial seawater) [34] was selected to eliminate the intensive neutral lipid accumulation [22]. The cells were cultured with the initial cell concentration of $1.0 \times 10^{6} \mathrm{cells} \cdot \mathrm{mL}^{-1}$ in flat-shape flasks $(1.5 \mathrm{~L})$. The growth curve was shown in Supplementary Figure S1. The temperature was maintained at $25 \pm 1{ }^{\circ} \mathrm{C}$, and continuous illumination was applied at $200 \mu \mathrm{mol} \cdot \mathrm{m}^{-2} \cdot \mathrm{s}^{-1}$. The cultures were bubbled with sterile air containing $2 \% \mathrm{CO}_{2}$ at a flow rate of $0.8 \mathrm{~L} / \mathrm{min}$. After $180 \mathrm{~h}$ of cultivation, the cells were harvested by centrifugation at $8500 \times g$ for $15 \mathrm{~min}$ and freeze-dried. 


\subsection{Lipid Extraction}

Total lipids were extracted from the lyophilized cells by two-step chloroform/methanol extraction described by Ejsing et al. [35] with some modifications. In general, $25 \mathrm{mg}$ of the lyophilized microalgal cells were re-suspended in $5 \mathrm{~mL}$ of chloroform/methanol $(20: 1 \mathrm{v} / \mathrm{v})$ and disrupted by 15 min-sonication. Phase separation was induced by adding distillated water $(1 \mathrm{~mL})$. After centrifugation at $800 \times \mathrm{g}$ for $10 \mathrm{~min}$, the lower phase was collected. The remaining aqueous sample material was processed with the extraction procedure again with $5 \mathrm{~mL}$ chloroform/methanol (2:1, v/v). The lower organic phase was collected and combined with the formerly collected organic phase. The lipid extracts were dried under argon gas and stored at $-30{ }^{\circ} \mathrm{C}$.

\subsection{Lipid Fractionation}

In order to avoid the unwanted overlap of isotopic ion peaks in the direct infusion ESI-Q-TRAP MS analysis, the solid phase extraction (SPE) was carried out to separate the total lipid extract. The Supelclean $^{\mathrm{TM}}$ LC-Si SPE Tube $(100 \mathrm{mg}, 1 \mathrm{~mL})$ was used to fractionate the total lipids into neutral lipids (NLs), glycolipids (GLs), and phospholipids (PLs) according to the method of Popovich et al. [36] with minor modifications. The columns were conditioned with $4 \mathrm{~mL}$ of hexane. The crude lipid extracts were dissolved in $200 \mu \mathrm{L}$ of chloroform and loaded into the column. NLs were eluted with $4 \mathrm{~mL}$ of chloroform, and GLs were eluted with $4 \mathrm{~mL}$ of acetone/methanol (9:1 v/v), finally PLs were eluted with $4 \mathrm{~mL}$ of methanol. Each lipid fraction was collected into a glass vial and dried under argon gas. The efficiency of SPE separation was verified by thin-layer chromatography (TLC). Briefly, each lipid fraction was analyzed by TLC. The neutral lipids were developed by $n$-hexane:diethyl ether:acetic acid 70:30:1 (v/v/v), and the polar lipis were developed by chloroform:methanol:acetic acid:water 170:30:20:7 (v/v/v/v). Neutral lipids and polar lipids were stained with iodine, while glycolipids were stained with orcinol. No contaminate lipid bands were observed in the lane of each lipid fraction on the TLC plates (data not shown).

\subsection{Direct Infusion Tandem MS/MS Analysis}

PL and GL fractions were re-dissolved in chloroform/methanol $(2: 1 \mathrm{v} / \mathrm{v})$. Adequate amount of lipid samples were added into the corresponding solvents according to the detecting ion mode. Chloroform/methanol (2:1 v/v with $1 \%$ of formic acid) was for the detection at positive ion mode, and chloroform/methanol $(1: 2 \mathrm{v} / \mathrm{v})$ was for the detection at negative ion mode. Samples were introduced into ESI-Q-TRAP-MS (QTRAP4000, AB Sciex) with direct infusion at the flow rate of $10 \mu \mathrm{L} / \mathrm{min}$. Sulfoquinovosyldiacylglycerols (SQDGs) were detected by Q1 scan at negative ion mode, while monogalactosyldiacylglycerols (MGDGs) and digalactosyldiacylglyerols (DGDGs) were detected by neutral loss scan at positive ion mode for the loss of $\mathrm{m} / \mathrm{z} 162$ which corresponding to the loss of a sugar group (galactose). Phosphatidylcholines (PCs) were detected by precursor ion scan for 184.07 (phosphocholine group) at positive ion modes, while other PLs including phosphatidylglycerols (PGs) and Phosphatidylinositos (PIs) were detected by Q1 scan at negative ion mode. Lipids were determined with their calculated $\mathrm{m} / \mathrm{z}$. The fatty acid composition and positional distribution $[37,38]$ were identified 
with product ion scan of each lipid molecule. Generally, the center position of the glycerol backbone is referred to as $s n-2$ position, and other ends are $s n-1$ and sn- 3 positions.

\section{Conclusions}

In conclusion, the polar lipid profile in Fistulifera solaris JPCC DA0580 was determined with direct infusion ESI-Q-TRAP-MS and MS/MS. Based on our analysis, the chloroplast was demonstrated to play an essential role in the lipid biosynthesis in $F$. solaris. EPA was synthesized on PC in $F$. solaris. In addition, we proposed that the PC-based acyl-editing and head group exchange processes may contribute to the incorporation of EPA into TAGs. To our best knowledge, this is the first report showing the possibility that the PC-based acyl-editing and head group exchange could contribute to the EPA-incorporation into TAG in diatoms. According to this study, the key enzymes in the acyl-editing and head group exchange processes, the PLA, LPCAT and PDCT, can be the future targets for metabolic engineering towards the regulation of EPA content for different purpose.

\section{Acknowledgments}

This study was supported by Japan Science and Technology Agency (JST), Core Research for Evolutionary Science and Technology (CREST).

\section{Author Contributions}

Tsuyoshi Tanaka designed overall experiments. Yue Liang performed experiments and analyzed data. All authors, including Yue Liang, Yoshiaki Maeda, Tomoko Yoshino, Mitsufumi Matsumoto, and Tsuyoshi Tanaka participated in discussions and preparation of the manuscript.

\section{Conflicts of Interest}

The authors declare no conflict of interest.

\section{References}

1. Hu, Q.; Sommerfeld, M.; Jarvis, E.; Ghirardi, M.; Posewitz, M.; Seibert, M.; Darzins, A. Microalgal triacylglycerols as feedstocks for biofuel production: Perspectives and advances. Plant J. 2008, 54, 621-639.

2. Li, Y.; Horsman, M.; Wu, N.; Lan, C.Q.; Dubois-Calero, N. Biofuels from microalgae. Biotechnol. Prog. 2008, 24, 815-820.

3. Lam, M.K.; Lee, K.T. Microalgae biofuels: A critical review of issues, problems and the way forward. Biotechnol. Adv. 2012, 30, 673-690.

4. Liang, Y.; Sarkany, N.; Cui, Y. Biomass and lipid productivities of Chlorella vulgaris under autotrophic, heterotrophic and mixotrophic growth conditions. Biotechnol. Lett. 2009, 31, 1043-1049.

5. Rodolfi, L.; Chini Zittelli, G.; Bassi, N.; Padovani, G.; Biondi, N.; Bonini, G.; Tredici, M.R. Microalgae for oil: Strain selection, induction of lipid synthesis and outdoor mass cultivation in a low-cost photobioreactor. Biotechnol. Bioeng. 2009, 102, 100-112. 
6. Mata, T.M.; Martins, A.A.; Caetano, N.S. Microalgae for biodiesel production and other applications: A review. Renew. Sustain. Energy Rev. 2010, 14, 217-232.

7. Radakovits, R.; Jinkerson, R.E.; Fuerstenberg, S.I.; Tae, H.; Settlage, R.E.; Boore, J.L.; Posewitz, M.C. Draft genome sequence and genetic transformation of the oleaginous alga Nannochloropis gaditana. Nat. Commun. 2012, 3, doi:10.1038/ncomms1688.

8. Chiu, S.-Y.; Kao, C.-Y.; Tsai, M.-T.; Ong, S.-C.; Chen, C.-H.; Lin, C.-S. Lipid accumulation and $\mathrm{CO}_{2}$ utilization of Nannochloropsis oculata in response to $\mathrm{CO}_{2}$ aeration. Bioresour. Technol. 2009, 100, 833-838.

9. Satoh, A.; Ichii, K.; Matsumoto, M.; Kubota, C.; Nemoto, M.; Tanaka, M.; Yoshino, T.; Matsunaga, T.; Tanaka, T. A process design and productivity evaluation for oil production by indoor mass cultivation of a marine diatom, Fistulifera sp. JPCC DA0580. Bioresour. Technol. 2013, 137, 132-138.

10. Knothe, G. Dependence of biodiesel fuel properties on the structure of fatty acid alkyl esters. Fuel Proc. Technol. 2005, 86, 1059-1070.

11. Chisti, Y. Biodiesel from microalgae. Biotechnol. Adv. 2007, 25, 294-306.

12. Deckelbaum, R.J.; Torrejon, C. The omega-3 fatty acid nutritional landscape: Health benefits and sources. J. Nutr. 2012, 142, 587S-591S.

13. Arao, T.; Kawaguchi, A.; Yamada, M. Positional distribution of fatty acids in lipids of the marine diatom Phaeodactylum tricornutum. Phytochemistry 1987, 26, 2573-2576.

14. Arao, T.; Yamada, M. Biosynthesis of polyunsaturated fatty acids in the marine diatom, Phaeodactylum tricornutum. Phytochemistry 1994, 35, 1177-1181.

15. Arao, T.; Sakaki, T.; Yamada, M. Biosynthesis of polyunsaturated lipids in the diatom, Phaeodactylum tricornutum. Phytochemistry 1994, 36, 629-635.

16. Alonso, D.L.; Belarbi, E.-H.; Fernández-Sevilla, J.M.; Rodríguez-Ruiz, J.; Grima, E.M. Acyl lipid composition variation related to culture age and nitrogen concentration in continuous culture of the microalga Phaeodactylum tricornutum. Phytochemistry 2000, 54, 461-471.

17. Bowler, C.; Allen, A.E.; Badger, J.H.; Grimwood, J.; Jabbari, K.; Kuo, A.; Maheswari, U.; Martens, C.; Maumus, F.; Otillar, R.P.; et al. The Phaeodactylum genome reveals the evolutionary history of diatom genomes. Nature 2008, 456, 239-244.

18. Miyahara, M.; Aoi, M.; Inoue-Kashino, N.; Kashino, Y.; Ifuku, K. Highly efficient transformation of the diatom Phaeodactylum tricornutum by multi-pulse electroporation. Biosci. Biotechnol. Biochem. 2013, 77, 874-876.

19. Apt, K.E.; Kroth-Pancic, P.G.; Grossman, A.R. Stable nuclear transformation of the diatom Phaeodactylum tricornutum. Mol. Gen. Genet. 1996, 252, 572-579.

20. Matsumoto, M.; Mayama, S.; Nemoto, M.; Fukuda, Y.; Muto, M.; Yoshino, T.; Matsunaga, T.; Tanaka, T. Morphological and molecular phylogenetic analysis of the high triglyceride-producing marine diatom, Fistulifera solaris sp. nov. (Bacillariophyceae). Phycol. Res. 2014, in press.

21. Matsumoto, M.; Sugiyama, H.; Maeda, Y.; Sato, R.; Tanaka, T.; Matsunaga, T. Marine diatom, Navicula sp. strain JPCC DA0580 and marine green alga, Chlorella sp. strain NKG400014 as potential sources for biodiesel production. App. Biochem. Biotechnol. 2010, 161, 483-490. 
22. Liang, Y.; Maeda, Y.; Matsumoto, M.; Yoshino, T.; Tanaka, T. Profiling of fatty acid methyl esters from the oleaginous diatom Fistulifera sp. strain JPCC DA0580 under nutrition-sufficient and -deficient conditions. J. Appl. Phycol. 2014, doi:10.1007/s10811-014-0265-y.

23. Sato, R.; Maeda, Y.; Yoshino, T.; Tanaka, T.; Matsumoto, M. Seasonal variation of biomass and oil production of the oleaginous diatom Fistulifera sp. in outdoor vertical bubble column and raceway-type bioreactors. J. Biosci. Bioeng. 2014, 117, 720-724.

24. Tanaka, T.; Fukuda, Y.; Yoshino, T.; Maeda, Y.; Muto, M.; Matsumoto, M.; Mayama, S.; Matsunaga, T. High-throughput pyrosequencing of the chloroplast genome of a highly neutral-lipid-producing marine pennate diatom, Fistulifera sp. strain JPCC DA0580. Photosynth. Res. 2011, 109, 223-229.

25. Muto, M.; Fukuda, Y.; Nemoto, M.; Yoshino, T.; Matsunaga, T.; Tanaka, T. Establishment of a genetic transformation system for the marine pennate diatom Fistulifera sp. strain JPCC DA0580 - A high triglyceride producer. Mar. Biotechnol. 2013, 15, 48-55.

26. Liang, Y.; Maeda, Y.; Sunaga, Y.; Muto, M.; Matsumoto, M.; Yoshino, T.; Tanaka, T. Biosynthesis of polyunsaturated fatty acids in the oleaginous marine diatom Fistulifera sp. strain JPCC DA0580. Mar. Drugs 2013, 11, 5008-5023.

27. Somerville, C. Plant lipids: Metabolism, mutants, and membranes. Science 1991, 252, 80-87.

28. Fan, J.; Andre, C.; Xu, C. A chloroplast pathway for the de novo biosynthesis of triacylglycerol in Chlamydomonas reinhardtii. FEBS Lett. 2011, 585, 1985-1991.

29. Yongmanitchai, W.; Ward, O.P. Molecular species of triacylglycerols from the freshwater diatom, Phaeodactylum tricor. Phytochemistry 1993, 32, 1137-1139.

30. Yongmanitchai, W.; Ward, O.P. Positional distribution of fatty acids, and molecular species of polar lipids, in the diatom Phaeodactylum tricornutum. J. Gen. Microbiol. 1993, 139, 465-472.

31. Muto, M.; Kubota, C.; Tanaka, M.; Satoh, A.; Matsumoto, M.; Yoshino, T.; Tanaka, T. Identification and functional analysis of delta-9 desaturase, a key enzyme in PUFA synthesis, isolated from the oleaginous diatom Fistulifera. PLoS One 2013, 8, e73507.

32. Bates, P.D.; Browse, J. The significance of different diacylgycerol synthesis pathways on plant oil composition and bioengineering. Front. Plant Sci. 2012, 3, doi:10.3389/fpls.2012.00147.

33. Bates, P.D.; Fatihi, A.; Snapp, A.R.; Carlsson, A.S.; Lu, C. Acyl editing and headgroup exchange are the major mechanisms that direct polyunsaturated fatty acid flux into triacylglycerols. Plant Physiol. 2012, 160, 1530-1539.

34. Guillard, R.R.; Ryther, J.H. Studies of marine planktonic diatoms: I. Cyclotella Nana Hustedt, and Detonula Confervacea (Cleve) Gran. Can. J. Microbiol. 1962, 8, 229-239.

35. Ejsing, C.S.; Sampaio, J.L.; Surendranath, V.; Duchoslav, E.; Ekroos, K.; Klemm, R.W.; Simons, K.; Shevchenko, A. Global analysis of the yeast lipidome by quantitative shotgun mass spectrometry. Proc. Natl. Acad. Sci. USA 2009, 106, 2136-2141.

36. Popovich, C.A.; Damiani, C.; Constenla, D.; Leonardi, P.I. Lipid quality of the diatoms Skeletonema costatum and Navicula gregaria from the South Atlantic Coast (Argentina): Evaluation of its suitability as biodiesel feedstock. J. Appl. Phycol. 2012, 24, 1-10.

37. Malone, M.; Evans, J.J. Determining the relative amounts of positional isomers in complex mixtures of triglycerides using reversed-phase high-performance liquid chromatography-tandem mass spectrometry. Lipids 2004, 39, 273-284. 
38. Xu, J.; Chen, D.; Yan, X.; Chen, J.; Zhou, C. Global characterization of the photosynthetic glycerolipids from a marine diatom Stephanodiscus sp. by ultra performance liquid chromatography coupled with electrospray ionization-quadrupole-time of flight mass spectrometry. Anal. Chim. Acta 2010, 663, 60-68.

(C) 2014 by the authors; licensee MDPI, Basel, Switzerland. This article is an open access article distributed under the terms and conditions of the Creative Commons Attribution license (http://creativecommons.org/licenses/by/3.0/). 